\title{
Consistent data-driven computational mechanics
}

D. González, F. Chinesta, and E. Cueto

Citation: AIP Conference Proceedings 1960, 090005 (2018); doi: 10.1063/1.5034931

View online: https://doi.org/10.1063/1.5034931

View Table of Contents: http://aip.scitation.org/toc/apc/1960/1

Published by the American Institute of Physics

\section{Articles you may be interested in}

Data-driven in computational plasticity

AIP Conference Proceedings 1960, 090006 (2018); 10.1063/1.5034932

Model and system learners, optimal process constructors and kinetic theory-based goal-oriented design: A new paradigm in materials and processes informatics

AIP Conference Proceedings 1960, 090004 (2018); 10.1063/1.5034930

Prediction of composites behavior undergoing an ATP process through data-mining

AIP Conference Proceedings 1960, 020016 (2018); 10.1063/1.5034817

A manifold learning approach to data-driven computational materials and processes

AIP Conference Proceedings 1896, 170003 (2017); 10.1063/1.5008201

A cyber physical system approach for composite part: From smart manufacturing to predictive maintenance AIP Conference Proceedings 1960, 020025 (2018); 10.1063/1.5034826

Simulation of the microwave heating of a thin multilayered composite material: A parameter analysis

AIP Conference Proceedings 1960, 020035 (2018); 10.1063/1.5034836 


\title{
Consistent Data-Driven Computational Mechanics
}

\author{
D. González ${ }^{1, a)}$, F. Chinesta ${ }^{2, \text { b) }}$ and E. Cueto ${ }^{1, c)}$ \\ ${ }^{1}$ Aragon Institute of Engineering Research. Universidad de Zaragoza. Zaragoza, Spain. \\ ${ }^{2}$ ESI Chair and PIMM lab. ENSAM ParisTech. Paris, France. \\ a) gonzal@unizar.es \\ b) francisco.chinesta@ensam.eu \\ ${ }^{c)}$ Corresponding author: ecueto@unizar.es
}

\begin{abstract}
We present a novel method, within the realm of data-driven computational mechanics, to obtain reliable and thermodynamically sound simulation from experimental data. We thus avoid the need to fit any phenomenological model in the construction of the simulation model. This kind of techniques opens unprecedented possibilities in the framework of data-driven application systems and, particularly, in the paradigm of industry 4.0.
\end{abstract}

\section{INTRODUCTION}

In recent years an increasing interest on the development of methods for data-driven simulation has arose. These methods eliminate the need for phenomenological constitutive equation fitting, a process that is frequently cumbersome. Instead, they work operating solely on experimental data [1, 2, 3, 4]. However, the need to guarantee thermodynamic consistency continues to be an issue for these methods. Since in general any experimental result will incorporate errors or some level of experimental noise, a doubt is frequently expressed in terms of the need to guarantee the satisfaction of basic principles. These include the laws of thermodynamics, notably.

To overcome these limitations, in a recent work, we have proposed to formulate the problem in the so-called GENERIC formalism [5, 6, 7]. The GENERIC ("General Equation for Non-Equilibrium Reversible-Irreversible Coupling") formalism establishes a completely general equation of the dynamics of a system under reversible and irreversible conditions, as dictated by the evolution of energy and entropy, respectively.

The state space of the system will be described by judiciously chosen variables in a space $S$. Therefore, the system will be described at time $t \in I$ by a function $z_{t}=z(t): I \rightarrow S, z \in \mathcal{C}^{1}(0, T)$. The GENERIC structure of the evolution equations for such a system takes the form

$$
\dot{\mathbf{z}}=\boldsymbol{L}\left(\mathrm{z}_{t}\right) \boldsymbol{\nabla} E\left(\mathrm{z}_{t}\right)+\boldsymbol{M} \boldsymbol{\nabla} E\left(\mathrm{z}_{t}\right), \quad \mathbf{z}(0)=\mathbf{z}_{0} .
$$

This equation must be supplemented with the so-called "degeneracy conditions",

$$
\begin{gathered}
\boldsymbol{L}\left(\mathbf{z}_{t}\right) \cdot \boldsymbol{\nabla} S\left(\mathbf{z}_{t}\right)=\mathbf{0}, \\
\boldsymbol{M}\left(\mathbf{z}_{t}\right) \cdot \boldsymbol{\nabla} E\left(\mathbf{z}_{t}\right)=\mathbf{0} .
\end{gathered}
$$

By choosing L skew-symmetric and M symmetric, positive semi-definite, one also ensures the conservation of energy and the strict entropy dissipation.

\section{PROPOSED IDENTIFICATION ALGORITHM}

We assume to know a certain amount of experimental data in the form of a vector-valued set $Z=\left\{z_{0}, z_{1}, \ldots\right.$, $\left.z_{\text {nmeas }}\right\}$. By discretizing the GENERIC equation, one arrives at 


$$
\frac{\mathbf{z}_{n+1}-\mathbf{z}_{n}}{\Delta t}=L\left(\mathbf{z}_{n+1}, \mathbf{z}_{n}\right) D E\left(\mathbf{z}_{n+1}, \mathbf{z}_{n}\right)+M\left(\mathbf{z}_{n+1}, \mathbf{z}_{n}\right) D S\left(\mathbf{z}_{n+1}, \mathbf{z}_{n}\right)
$$

where $L$, and $M$ represent the discrete version of the Poisson and friction operators, $\boldsymbol{L}$ and $\boldsymbol{M}$, and $D E$ and $D S$ represent the discrete gradients, respectively. It is worth noting that, by identifying the particular form of $E(z)$ we are doing no other thing than identifying what we usually call the "constitutive law" in solid mechanics.

Therefore, in the just proposed data-driven identification algorithm we are solving a data assimilation problem of the form

$$
\boldsymbol{\mu}=\{L, M, D E, D S\}=\arg \min _{\boldsymbol{\mu} *}\left\|\mathbf{z}(\boldsymbol{\mu})-\mathbf{z}^{\text {meas }}\right\|,
$$

with $z^{\text {meas }}$ a subset of $Z$, the total number of experimental results.

\section{RESULTS}

\section{Simple pendulum}

The first problem corresponds to a simple pendulum whose purely Hamiltonian structure, in absence of any dissipation, responds to the case $\boldsymbol{M}=\mathbf{0}$. Therefore, only the discrete matrix form of the gradient $D E$ was identified.

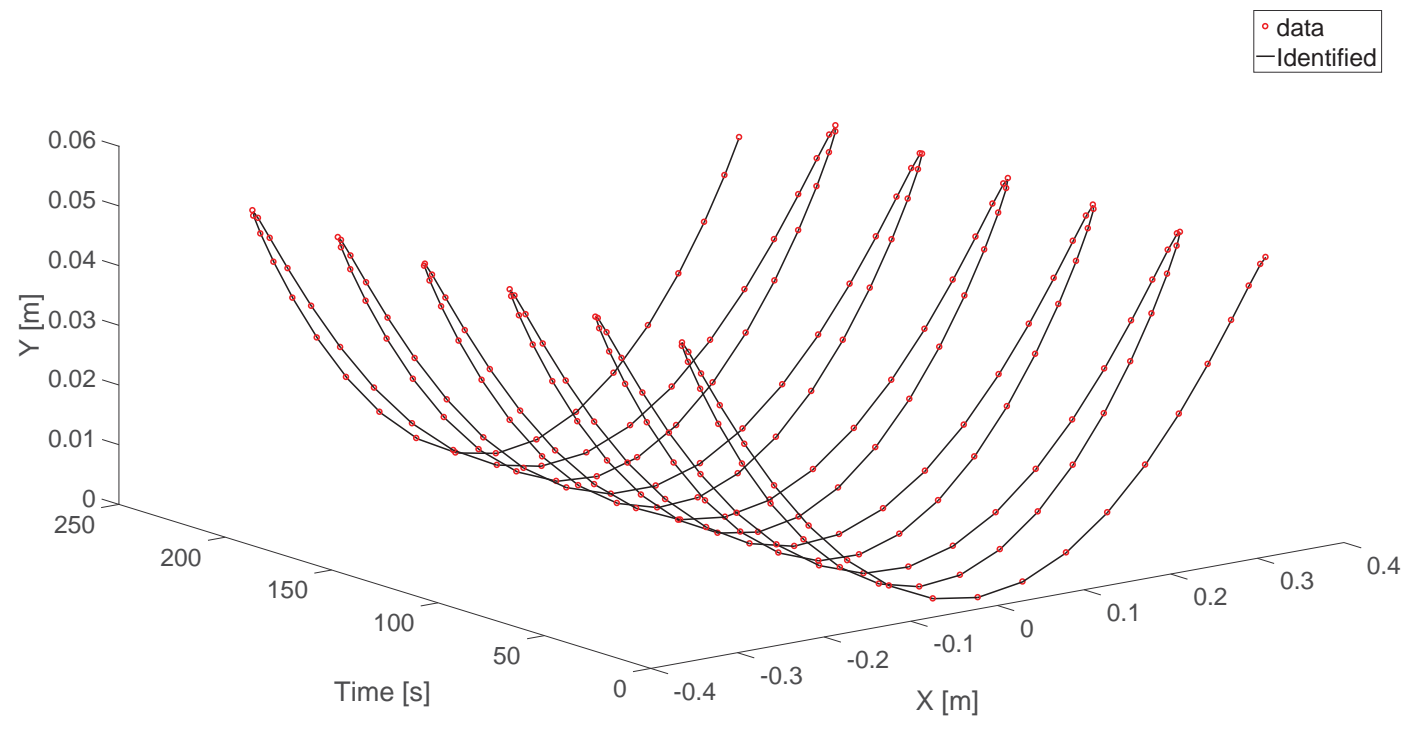

FIGURE 1. Results for the identification of the Hamiltonian structure of a simple pendulum.

In this case, the error in the identification procedure vanishes within machine precision, if we employ the whole set of 241 experimental measurements. By taking them in sub-sets of five experimental values, the error increases to $0.44 \%$, see Fig. 2. 


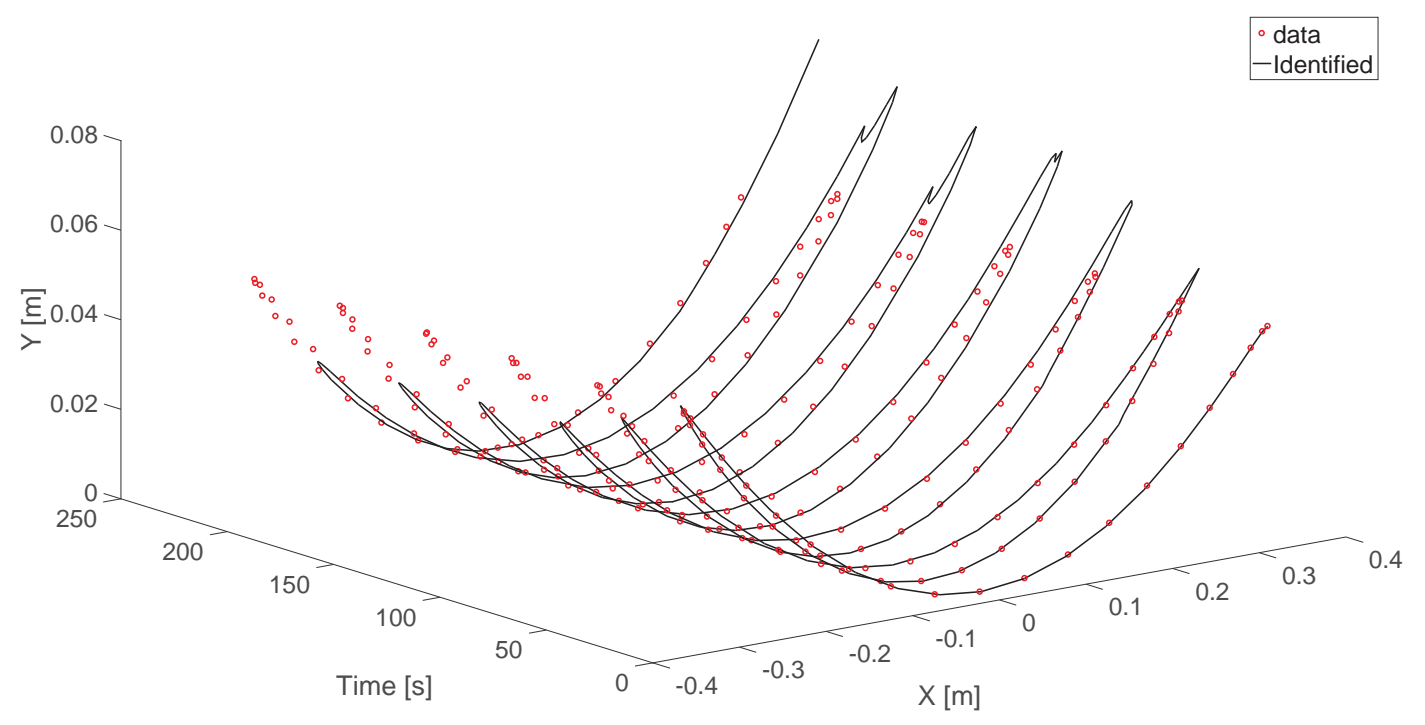

FIGURE 2. Results for the identification of the Hamiltonian structure of a simple pendulum. Data sets composed on only five temporal series.

\section{Thermoelastic pendulum}

As an example of non-equilibrium system, we have chosen the thermoelastic pendulum introduced in [8], see Fig. 3.

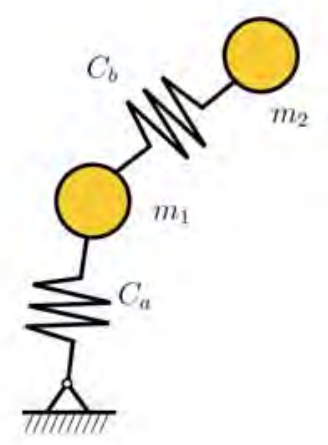

FIGURE 3. Thermoelastic pendulum.

The set of variables describing the pendulum will be given by

$$
\boldsymbol{S}=\left\{z=\left(\boldsymbol{q}_{1}, \boldsymbol{q}_{2}, \boldsymbol{p}_{1}, \boldsymbol{p}_{2}, s_{1}, s_{2}\right) \in\left(\mathfrak{R}^{2} \times \mathfrak{R}^{2} \times \mathfrak{R}^{2} \times \mathfrak{R}^{2} \times \mathfrak{R} \times \mathfrak{R}\right), \boldsymbol{q}_{1} \neq 0, \boldsymbol{q}_{2} \neq 0\right\},
$$

Where $\boldsymbol{q}$ and $\boldsymbol{p}$ represent position and momenta, respectively, and $s$ represents entropy. Results for the identification of this system are shown in Fig. 4. 


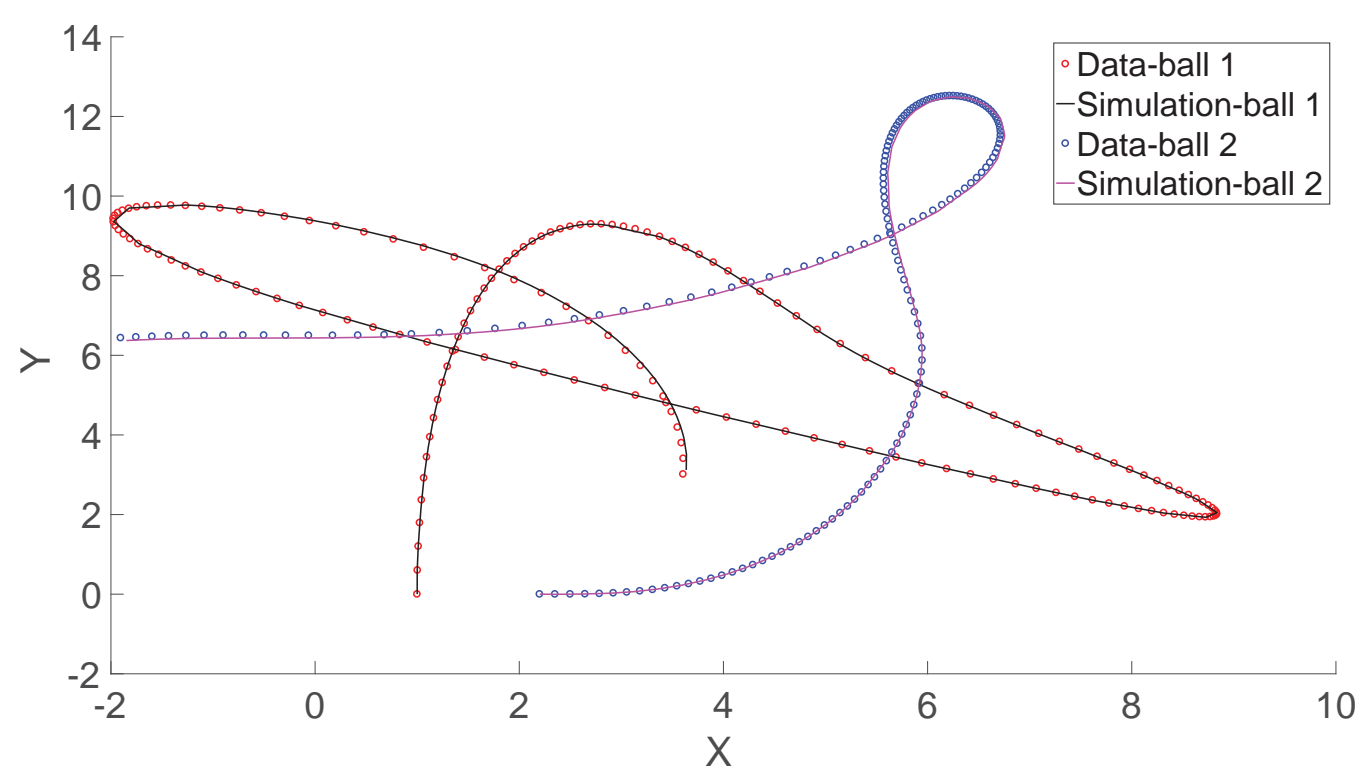

FIGURE 4. Results for the thermal pendulum problem. Experimental results were grouped in sets of 34 time instants.

\section{CONCLUSIONS}

The present approach is based on the usage of the GENERIC paradigm, a framework that guarantees the strict energy conservation and positive entropy production, along with the symmetries of the system, and that generalizes the Hamiltonian form of classical mechanics to non-equilibrium systems. The proposed methodology thus identifies the proper GENERIC structure of the problem. The method identifies either the whole GENERIC structure composed by the Poisson and friction matrices of the problem, plus the precise numerical form of the gradients of the energy and entropy. However, for some complex problems it has been found that the form of the Hamiltonian and dissipative structures of the problem is easy to identify, and that the method works considerably better by just finding the form of the gradients of energy and entropy, respectively. This is equivalent, of course, to finding in a numerical, point-wise manner, the form of the constitutive manifold that the authors defined in a previous work.

But the proposed methodology not only provides with a suitable method to unveil the precise form of the Hamiltonian and dissipative structures of the problem. It also provides a robust and thermodynamically consistent way to integrate these equations without employing any constitutive equation (the so-called data-driven computational mechanics paradigm).

\section{ACKNOWLEDGMENTS}

This work has been supported by the Spanish Ministry of Economy and Com- petitiveness through Grants number DPI2017-85139-C2-1-R and DPI2015- 72365-EXP and by the Regional Government of Aragon and the European Social Fund, research group T88.

\section{REFERENCES}

1. T. Kirchdoerfer and M. Ortiz. Data-driven computational mechanics. Computer Methods in Applied Mechanics and Engineering, 304:81 - 101, 2016. 
2. R. Ibañez, D. Borzacchiello, J. V. Aguado, E. Abisset-Chavanne, E. Cueto, P. Ladeveze, and F. Chinesta. Data-driven non-linear elastic- ity. constitutive manifold construction and problem discretization. Computational Mechanics, 60(5):813-826, 2017.

3. Ruben Ibanñez, Emmanuelle Abisset-Chavanne, Jose Vicente Aguado, David Gonzalez, Elias Cueto, and Francisco Chinesta. A manifold learn- ing approach to data-driven computational elasticity and inelasticity. Archives of Computational Methods in Engineering, 2018.

4. E. Lopez, D. Gonzalez, J. V. Aguado, E. Abisset-Chavanne, E. Cueto, C. Binetruy, and F. Chinesta. A manifold learning approach for inte- grated computational materials engineering. Archives of Computational Methods in Engineering, 2018.

5. Miroslav Grmela and Hans Christian Ötinger. Dynamics and thermo-dynamics of complex fluids. i. development of a general formalism. Phys. Rev. E, 56:6620-6632, Dec 1997.

6. H. C. Oettinger. Beyond Equilibrium Thermodynamics. Wiley, 2005.

7. Hans C. Ötinger. Non equilibrium thermodynamics: a powerful tool for scientists and engineers. DYNA, 79:122-128, 2012.

8. Ignacio Romero. Thermodynamically consistent time-stepping algo- rithms for non-linear thermomechanical systems. International Journal for Numerical Methods in Engineering, 79(6):706-732, 2009. 